\title{
Percepção sobre mídia e comportamento na compra de alimentos: estudo com consumidores de dois municípios do sul de Minas Gerais
}

\author{
Perceptions about the media and behaviour when buying food \\ products: case study with consumers from two cities in the \\ south of Minas Gerais State
}

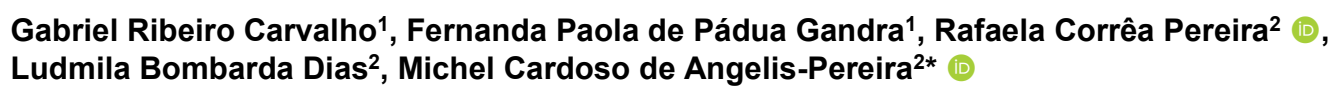

${ }^{1}$ Universidade Federal de Lavras (UFLA), Departamento de Ciência dos Alimentos, Lavras/MG - Brasil

'Universidade Federal de Lavras (UFLA), Departamento de Nutrição, Lavras/MG - Brasil

*Corresponding Author: Michel Cardoso de Angelis-Pereira, Universidade Federal de Lavras (UFLA), Departamento de Nutrição, Campus Universitário, CEP: 37200-000, Lavras/MG - Brasil, e-mail: deangelis@dnu.ufla.br

Cite as: Carvalho, G. R., Gandra, F. P. P., Pereira, R. C., Dias, L. B., \& Angelis-Pereira, M. C. (2019). Perceptions about the media and behaviour when buying food products: case study with consumers from two cities in the south of Minas Gerais State. Brazilian Journal of Food Technology, 22, e2018170. https://doi.org/10.1590/19816723.17018

\begin{abstract}
Resumo
A mídia e as propagandas de alimentos representam hoje importantes estratégias para diferenciação, promoção e venda de produtos pelas indústrias. Dessa forma, estas podem exercer forte influência sobre o comportamento de compra e consumo do consumidor. Diante destas considerações, o objetivo deste trabalho foi avaliar a percepção de consumidores sobre a mídia e suas influências sobre o comportamento de compra de alimentos, por meio de uma pesquisa de mercado com consumidores de dois municípios do sul do estado de Minas Gerais, Brasil. Foi usada uma amostra não probabilística por conveniência, totalizando 285 respondentes (150 em Varginha e 135 em Lavras), tendo como instrumento de pesquisa questões para entrevista elaboradas pelos próprios pesquisadores. Os dados foram analisados de forma descritiva. Diante dos resultados, pode-se evidenciar que, para a amostra em estudo, 0 consumo alimentar pode ser influenciado pelas mídias e pelas propagandas de alimentos nelas anunciadas. A exposição a estes anúncios também pode estar relacionada com as opiniões sobre o que mais se valoriza na compra de alimentos, como a praticidade e a conveniência. No que se refere à leitura dos rótulos, observou-se baixa frequência de consumidores que leem frequentemente estas informações. Juntos, estes resultados reforçam as evidências de que as estratégias de marketing têm o potencial de influenciar no comportamento alimentar dos sujeitos e, portanto, também contribuir para o alcance dos objetivos da saúde pública, no que tange a alimentação e nutrição. Ressalta-se, neste caso, a importância de estratégias de educação alimentar e nutricional para a formação de consumidores mais autônomos e críticos quanto às suas escolhas alimentares.
\end{abstract}

Palavras-chave: Saúde pública; Rótulo nutricional; Comportamento do consumidor; Indústria de alimentos; Marketing; Meios de comunicação; Alimentação. 


\begin{abstract}
Media and food advertisements today represent important industrial strategies for the differentiation, promotion and selling of products. Thus, they can exert a strong influence on consumer buying and consumption behaviour. Considering this, the objective of this work was to evaluate the perception of consumers about the media and its influence on food purchasing behaviour through a market research survey with consumers from two municipalities in the south of Minas Gerais State, Brazil. A non-probabilistic sample was used for convenience, totalizing 285 respondents (150 in Varginha and 135 in Lavras), and an interview with questions prepared by the researchers themselves being used as the research instrument. The data were analysed in a descriptive way. Based on the results, it could be seen that, for the sample under study, food consumption could be influenced by the media and by the food advertisements. Exposure to these ads may also be related to opinions about what is most valued in the purchase of food, such as practicality and convenience. Regarding the reading of labels, a low frequency of consumers who frequently read this information was observed. Together, these results reinforce the evidence that marketing strategies have the potential to influence the eating behaviour of the subjects, and, therefore, also contribute to the achievement of public health goals for food and nutrition. In this case, the importance of food and nutritional education strategies for the training of more autonomous and critical consumers regarding their food choices is highlighted.
\end{abstract}

Keywords: Public health; Nutritional label; Consumer behaviour; Food industry; Marketing; Communications media; Feeding.

\title{
1 Introdução
}

O estudo da nutrição e da alimentação adequada, bem como dos fatores interferentes na adoção de padrões alimentares e dietéticos pela população, têm sido o foco de muitas pesquisas nas áreas da ciência dos alimentos e de saúde pública. Isso decorre do contexto alimentar e de saúde da população na atualidade, que se caracteriza pelos altos índices de obesidade e doenças crônicas não transmissíveis (DCNT), somado às circunstâncias desfavoráveis dos sistemas produtivos alimentares modernos.

A rápida transformação observada na sociedade contemporânea, no que tange aos hábitos alimentares dos sujeitos, levou à proposição da teoria da transição nutricional. Esta teoria sugere que, em decorrência do desenvolvimento econômico das populações, se observou a substituição de dietas minimamente processadas, abundantes em alimentos de origem vegetal, para dietas ricas em carne, óleos e gorduras, e alimentos processados (Kelly, 2016).

A profusão de alimentos industrializados no mercado mundial criou ambiente competitivo, em que o marketing se torna essencial para o sucesso das companhias (Mayhew et al., 2016). Assim, os rótulos de alimentos, juntamente com a mídia, passaram a ser importantes ferramentas de marketing e não apenas recurso informativo, tornando-se, assim, meio estratégico para que a indústria exerça influência sobre o comportamento de compra e consumo do consumidor (Nestle \& Ludwig, 2010).

Diante destas considerações, o objetivo deste trabalho foi avaliar a percepção de consumidores sobre a mídia e o comportamento de compra dos alimentos, em dois municípios do sul de Minas Gerais, Brasil.

\section{Métodos}

\subsection{Amostragem}

A coleta dos dados da pesquisa foi realizada em pontos públicos de diferentes localizações geográficas dos municípios de Lavras e Varginha, no estado de Minas Gerais, Brasil. 
Ambas as cidades pesquisadas estão localizadas no sul de Minas Gerais e com Índice de Desenvolvimento Humano (IDH) superior a 0,75. O IDH é uma medida resumida do progresso em longo prazo nas três dimensões básicas do desenvolvimento humano: renda, educação e saúde (Programa das Nações Unidas para o Desenvolvimento, 2017). Lavras e Varginha contavam com uma população aproximada de 101 mil e 131 mil habitantes, respectivamente, em 2014 (Instituto Brasileiro de Geografia e Estatística, 2014).

Com o intuito de representar as populações das duas cidades, foi usada uma amostra não probabilística por conveniência, totalizando 285 respondentes (150 em Varginha e $135 \mathrm{em}$ Lavras). Este número foi considerado como adequado para representar as duas populações e foi calculado considerando o número total de indivíduos de cada população (número de habitantes de cada cidade), com intervalo de confiança de $95 \%$ e margem de erro de $10 \%$ (Ferreira, 2009).

\subsection{Roteiro da entrevista}

O instrumento utilizado para a coleta dos dados foi uma entrevista elaborada pelos próprios pesquisadores, que constou de variáveis sociodemográficas, como: sexo, idade, grau de escolaridade, renda, principal responsável pela compra dos alimentos (5 questões - seção 1); questões sobre a relação do entrevistado com a mídia, como: tempo gasto, confiança, tipo de mídia mais acompanhado (3 questões - seção 2); questões sobre a relação da alimentação com o marketing e a frequência de leitura do rótulo, com perguntas sobre a influência que a mídia exerce nas escolhas por alimentos, se essa influência é percebida como negativa ou positiva, se sente atraído pelo alimento durante sua propaganda e se compra algum produto pela aparência de sua embalagem/rótulo (4 questões - seção 3), e questões sobre quais itens valoriza na hora da compra e frequência de leitura dos rótulos de produtos alimentícios (2 questões - seção 4). A entrevista apresentava um total de 14 perguntas.

A entrevista foi aplicada nos períodos matutino e vespertino, em pontos distintos das duas cidades, sob as mesmas orientações, sendo esclarecido aos pesquisados sobre o tempo das perguntas e o objetivo do trabalho antes de serem questionados. Durante a abordagem, o entrevistado foi esclarecido acerca de algumas circunstâncias: caso não entendesse alguma questão, a mesma seria repetida; havia a opção de não responder questões, e a possibilidade de parar a entrevista a qualquer momento.

\subsection{Análise dos resultados}

Os dados foram analisados de forma descritiva com auxílio do software SPSS ${ }^{\circledR}$ v. 11.0. Os resultados foram expressos em frequência relativa de respostas obtidas em cada questão de forma gráfica ou em tabelas.

\section{Resultados e discussão}

As características sociodemográficas das duas amostras em estudo indicaram que a maior parte dos respondentes tinha idade entre 21 e 35 anos (47\% em Lavras e 42\% em Varginha). Em Lavras, a maioria tinha como maior grau de escolaridade o Ensino Médio, enquanto, em Varginha, o Ensino Superior. A faixa de renda entre menos que 1 salário mínimo até 3 salários mínimos foi a que obteve maior percentual nas duas cidades (46\% em Lavras e 49\% em Varginha). Os que ganham mais de 10 salários mínimos representavam $5 \%$ em Lavras e $4 \%$ em Varginha (Tabela 1).

Tabela 1. Quantidade de pessoas entrevistadas de acordo com renda mensal.

\begin{tabular}{ccc}
\hline Renda (salários mínimos) & Lavras & Varginha \\
\hline Até 1 & 11 & 10 \\
Entre 1 e 3 & 52 & 64 \\
Entre 3 e 10 & 51 & 30 \\
Mais que 10 & 7 & 6 \\
Sem resposta & 14 & 40 \\
\hline
\end{tabular}


Entre os entrevistados, a maior parte afirmou ser o principal responsável pela compra dos alimentos que seu núcleo de convivência consome, em ambos os municípios (62\% em Lavras e 56\% em Varginha), tendo, assim, maior liberdade para adquirir os alimentos que deseja.

Os entrevistados foram questionados sobre o tipo de mídia que mais acompanha e o tempo diário gasto neste acompanhamento. Os resultados mostraram que a televisão foi a mídia mais acompanhada $(31 \% \mathrm{em}$ Lavras e $36 \%$ em Varginha), seguida pela internet (29\% em Lavras e Varginha). Em ambas as cidades, a maior parte dos entrevistados afirmou passar entre 1 e 3 horas do dia acompanhando as mídias em geral. Os resultados do grau de confiança que as mídias comunicam para amostra em estudo estão representados na Figura 1.

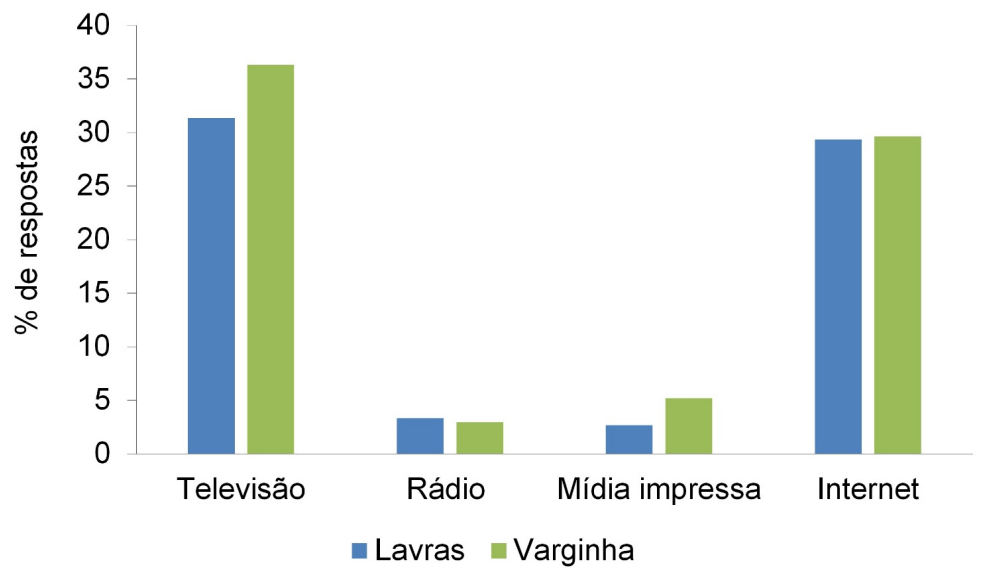

Figura 1. Tipos de mídia influenciadora no consumo de alimentos industrializados, de dois municípios do sul de Minas Gerais (Brasil).

Em ambas as cidades, a maior parte dos entrevistados classificou sua confiança na mídia nacional como média. Apenas $1 \%$ dos entrevistados da cidade de Lavras e $4 \%$ da cidade de Varginha disseram ter total confiança.

Com a inserção das novas mídias em seu cotidiano, a população acostumou-se a acessar grande quantidade de conteúdos de forma quase instantânea. Isso proporcionou condições para a criação de um cenário no qual há muita informação ao mesmo tempo em que se constrói pouco conhecimento. Para muitos sujeitos, isso pode ser motivo de desconfiança, o que implica na necessidade de criticidade ao se avaliarem as diferentes fontes de informação existentes (Almeida, 2012).

Para todas as pessoas que afirmaram ter total confiança na mídia, a televisão foi citada como o veículo de comunicação mais acompanhado. Não houve relação direta entre grau de confiança na mídia e o tempo que o mesmo indivíduo a acompanha, em nenhuma das cidades. Dos que depositam muita ou total confiança, $78 \%$ acompanham a mídia mais de uma hora por dia, contra $86 \%$ do restante da população.

De todos os entrevistados, $60 \%$ em Lavras e $71 \%$ em Varginha afirmaram sentir algum tipo de influência das mídias em seus hábitos alimentares, sendo que 3\% e 7\% interpretam essa influência como negativa, nas cidades de Lavras e Varginha, respectivamente. Ademais, $85 \%$ dos lavrenses e $82 \%$ dos varginhenses já se sentiram atraídos por alimentos durante sua propaganda, sendo que em Lavras e Varginha, $85 \%$ e $63 \%$ dos respondentes, respectivamente, afirmaram já ter decidido comprar algum alimento pela aparência da embalagem ou do rótulo.

Segundo Crivelaro et al. (2006), a televisão é o meio de comunicação que tem maior influência sobre a população. Note-se, ainda, que a população adulta, que corresponde à maioria dos respondentes da presente pesquisa, tende a sofrer maior influência das propagandas de persuasão lógica (Cook et al., 2011), isto é, aquelas que usam algum tipo de estratégia para convencer e não seduzir. 
De fato, numerosos estudos evidenciaram que o marketing de alimentos, principalmente daqueles ricos em gorduras saturadas, sódio e açúcar, contribui para a adoção de padrões dietéticos inadequados. Boyland et al. (2016), por exemplo, em uma revisão sistemática e meta-análise de artigos que avaliaram o efeito da exposição à publicidade (pela televisão e Internet) sobre o consumo alimentar, concluíram que os 22 estudos considerados elegíveis pela revisão mostraram aumento no consumo alimentar de crianças - mas não de adultos - expostas à publicidade de alimentos.

Estudos do efeito que a publicidade de alimentos exerce sobre o consumo alimentar de adultos ainda são limitados, sendo as evidências obtidas até o momento insuficientes para se formularem conclusões mais concretas (Mills et al., 2013). Algumas hipóteses, no entanto, sugerem que adultos sejam mais críticos em relação às propagandas pela maior capacidade cognitiva quando comparados com crianças (Boyland et al., 2016).

Em outra revisão sistemática e meta-análise, foram reunidos estudos que avaliaram o efeito da publicidade de alimentos e bebidas não saudáveis sobre a preferência alimentar de crianças e adolescentes com idade entre 2 e 18 anos. Evidenciou-se que a publicidade destes produtos aumentou a ingestão de alimentos de maior densidade energética e nutricionalmente desbalanceados, além de interferir nas preferências alimentares durante ou logo depois à exposição aos anúncios (Sadeghirad et al., 2016).

$\mathrm{Na}$ Austrália, Sainsbury et al. (2017) relataram que a publicidade de alimentos nas estações de trem do país se referia, basicamente, a alimentos processados não saudáveis, destinadas ao público em geral, de diferentes faixas etárias. Os autores ressaltaram, neste caso, a inadequação dos sistemas de autorregulamentação em proteger o público da exposição a este tipo de publicidade.

Tendo em vista que a publicidade de alimentos e bebidas é uma das principais categorias de anúncios televisivos e que há predomínio da divulgação de alimentos ultraprocessados, em sua maioria não saudáveis (Maia et al., 2017), a exposição a estas mídias pode ser um dos fatores que influenciam na adoção de hábitos alimentares inadequados pela população. Este fato reforça a necessidade e a importância de ações regulamentadoras da publicidade de alimentos no Brasil.

Estes indícios corroboram com Caivano et al. (2017), que ressaltam que há muitas evidências de que os alimentos ultraprocessados colaboram para a epidemia de obesidade e DCNT. Os autores destacam ainda que, dentre os principais fatores que contribuíram para o aumento no consumo desses alimentos, estão as campanhas de marketing, por meio da utilização de estratégias de formação de opinião, como a exposição de campanhas maciças para a promoção desses produtos na mídia, como televisão e internet.

Os entrevistados foram questionados sobre qual item eles mais valorizavam na hora da compra de alimentos: preço, promoções, propaganda, informações, acessibilidade, local da compra, praticidade do produto, qualidade do produto e o quão saudável é o produto. Estes itens foram agrupados de acordo com os quatro pilares do marketing (preço, promoção, local de compra e produto em si) (Figura 2).

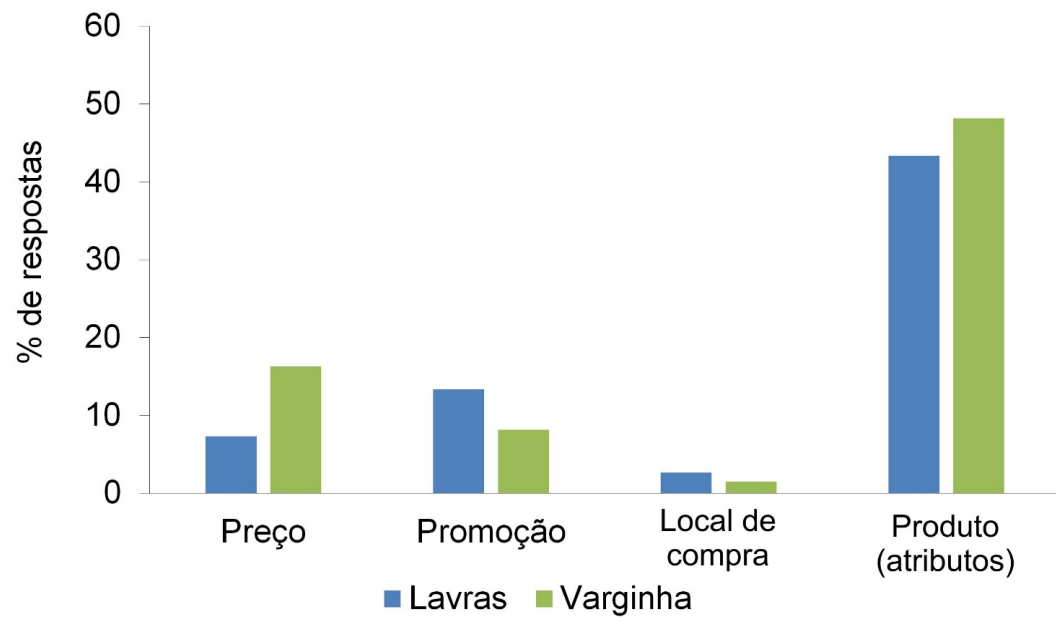

Figura 2. Grau de confiança que a mídia comunica para os indivíduos da amostra entrevistada, de dois municípios do sul de Minas Gerais (Brasil).

A atribuição para o produto em si foi identificada quando o entrevistado respondeu que o item mais valorizado seria a praticidade, a qualidade ou o quão saudável o produto seria ao realizar sua escolha. Tanto em Lavras como em Varginha, 65\% dos entrevistados disseram valorizar mais essas características ao 
comprar um alimento. Isso mostra que a maioria dos consumidores tem consciência de que a qualidade do produto em si é mais importante do que as promoções, o preço e o local no qual é vendido.

Com a profusão de alimentos industrializados nos mercados em nível mundial, criou-se ambiente competitivo, em que o marketing se torna essencial para o sucesso das companhias produtoras desses alimentos (Mayhew et al., 2016). Portanto, as campanhas de marketing colaboram para o aumento na aquisição de alimentos industrializados prontos para consumo, a partir da criação de necessidades no consumidor, da falta de tempo dedicada ao preparo dos alimentos, da comodidade, da praticidade e da conveniência trazidas por esses produtos (Caivano et al., 2017). Estas evidências podem explicar as respostas obtidas nesta pesquisa.

O resultado da frequência de leitura de rótulos de produtos alimentícios dos entrevistados é mostrado na Figura 3.

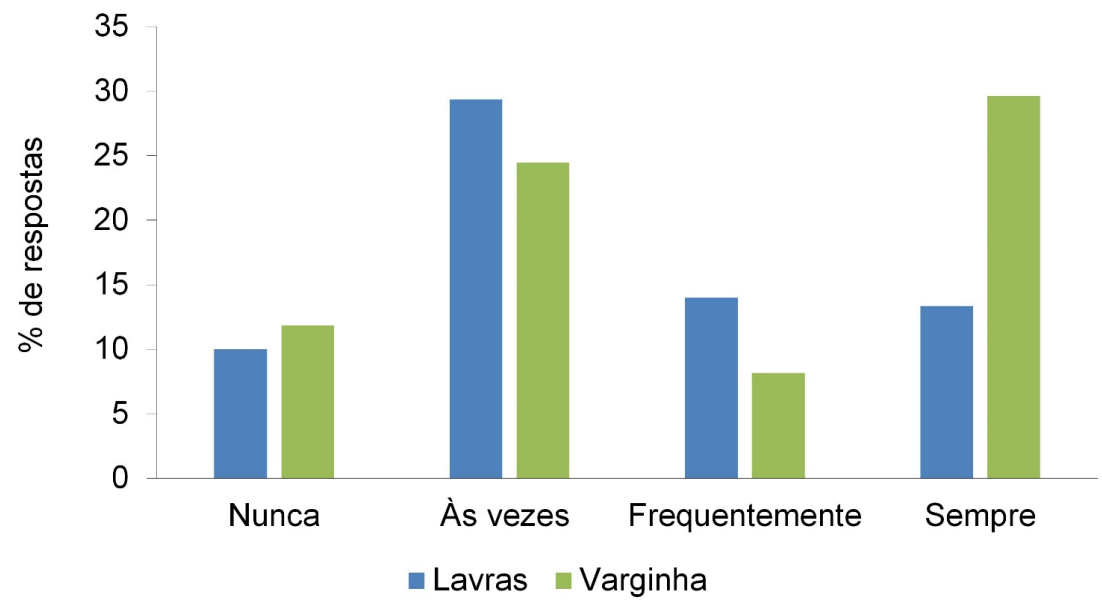

Figura 3. Atributos valorizados para escolha de alimentos industrializados por consumidores de dois municípios do sul de Minas Gerais (Brasil).

Observou-se que os entrevistados, $20 \%$ em Lavras e $40 \%$ em Varginha, disseram sempre ler rótulos antes de comprar um alimento. Por outro lado, 15\% dos respondentes de Lavras e 16\% dos de Varginha afirmaram nunca ler rótulos de produtos alimentícios.

Os respondentes com renda mensal de até um salário mínimo responderam que leem mais os rótulos do que aqueles que ganham mais de um salário. O resultado mostrou que, para estas populações, uma renda mensal mais elevada não está relacionada com maior frequência de leitura de rótulos.

Quanto ao grau de escolaridade, em Varginha, nenhum dos indivíduos que cursaram Ensino Superior ou pós-graduação relatou nunca ler rótulos antes das compras, diferente de Lavras, em que $8 \%$ das pessoas deste grupo afirmaram nunca ler.

Observou-se que as mulheres entrevistadas relataram maior frequência de leitura dos rótulos que os homens nas duas cidades. Em Lavras, $22 \%$ das mulheres disseram sempre ler os rótulos enquanto que $12 \%$ dos homens relataram a mesma frequência. Na cidade de Varginha, metade das mulheres afirmou sempre ler os rótulos diante de pouco mais de $30 \%$ dos homens.

As mulheres, por serem as principais responsáveis pelas compras da casa e por se interessarem mais por informações sobre alimentação e saúde do que os homens, tendem a ler com mais frequência as informações do rótulo (Cannoosamy et al., 2014; Hess et al., 2012; Drichoutis et al., 2006). Segundo Drichoutis et al. (2006), o grau de escolaridade também contribui para a maior frequência de leitura do rótulo, já que estes sujeitos tendem a entender melhor as informações apresentadas, usando-as para fazer suas escolhas durante a compra de alimentos. 
De fato, a leitura dos rótulos pode trazer informações fundamentais para que o consumidor possa fazer escolhas mais adequadas, contanto que tenha conhecimento suficiente para isto. Ademais, para que o consumidor possa utilizar esta ferramenta, é preciso que a veracidade das informações apresentadas pelo rótulo nutricional em alimentos seja garantida, cumprindo assim o objetivo de auxiliar o consumidor em suas escolhas. Entretanto, diversos estudos apontam não conformidades entre dados descritos na rotulagem nutricional e a real composição do alimento, fazendo com que o consumidor se sinta enganado (Domiciano et al., 2018; Marzarotto \& Alves, 2017).

\section{Conclusão}

Diante dos resultados desta pesquisa, pode-se observar que, para a amostra em estudo, os sujeitos entrevistados mostraram percepção de que o consumo alimentar pode ser influenciado pelas mídias e pelas propagandas de alimentos nelas anunciadas. A exposição a estes anúncios também pode estar relacionada com as opiniões sobre o que mais se valoriza na compra de alimentos, como a praticidade e a conveniência. No que se refere à leitura dos rótulos, observou-se baixa frequência de consumidores que leem frequentemente estas informações.

Juntos, estes resultados reforçam as evidências de que as estratégias de marketing têm o potencial de influenciar no comportamento alimentar dos sujeitos e, portanto, também contribuir para o alcance dos objetivos da saúde pública, no que tange a alimentação e nutrição. Ressalta-se, neste caso, a importância de estratégias de educação alimentar e nutricional para formação de consumidores mais autônomos e críticos quanto às suas escolhas alimentares.

\section{Referências}

Almeida, M. A. (2012). A promoção da saúde nas mídias sociais: Uma análise do perfil do Ministério da Saúde no Twitter (Trabalho de conclusão de curso). Universidade Federal de Goiás, Goiânia.

Boyland, E. J., Nolan, S., Kelly, B., Tudur-Smith, C., Jones, A., Halford, J. C., \& Robinson, E. (2016). Advertising as a cue to consume: A systematic review and meta-analysis of the effects of acute exposure to unhealthy food and nonalcoholic beverage advertising on intake in children and adults. The American Journal of Clinical Nutrition, 103(2), 519-533. PMid:26791177. http://dx.doi.org/10.3945/ajcn.115.120022

Caivano, S., Lopes, R. F., Sawaya, A. L., Domene, S. M. Á., \& Martins, P. A. (2017). Conflitos de interesses nas estratégias da indústria alimentícia para aumento do consumo de alimentos ultraprocessados e os efeitos sobre a saúde da população brasileira. DEMETRA: Alimentação, Nutrição \& Saúde, 12(2), 349-360. http://dx.doi.org/10.12957/demetra.2017.26928

Cannoosamy, K., Pugo-Gunsam, P., \& Jeewon, R. (2014). Consumer knowledge and attitudes toward nutritional labels. Journal of Nutrition Education and Behavior, 46(5), 334-340. PMid:24933235. http://dx.doi.org/10.1016/j.jneb.2014.03.010

Cook, I. A., Warren, C., Pajot, S. K., Schairer, D., \& Leuchter, A. F. (2011). Regional brain activation with advertising images. Journal of Neuroscience, Psychology, and Economics, 4(3), 147-160. http://dx.doi.org/10.1037/a0024809

Crivelaro, L. P., Sibinelli, E. C., \& Ibarra, J. A. (2006). A publicidade na TV e sua influência na obesidade infantil. UNIRevista, 1(3), 1-7.

Domiciano, C. G., Pereira, R. C., Picinin, C. T. R., Machado, F. S., \& De Angelis-Pereira, M. C. (2018). Food bar labels: Consumer behaviour and veracity of the available information. Brazilian Journal of Food Technology, 21(e2016131), http://dx.doi.org/10.1590/1981-6723.13116

Drichoutis, A. C., Lazaridis, P., \& Nayga, R. M. (2006). Consumers' use of nutritional labels: A review of research studies and issues. Journal of the Academy of Marketing Science, 9(9), 1-22.

Ferreira, D. F. (2009). Estatística básica. Lavras: UFLA.

Hess, R., Visschers, V. H., \& Siegrist, M. (2012). The role of health-related, motivational and sociodemographic aspects in predicting food label use: A comprehensive study. Public Health Nutrition, 15(3), 407-414. PMid:21752310. http://dx.doi.org/10.1017/S136898001100156X

Instituto Brasileiro de Geografia e Estatística - IBGE. (2014, agosto 28). Estimativas de população para $1^{\circ}$ de julho de 2014. Diário Oficial [da] República Federativa do Brasil, Brasília.

Kelly, M. (2016). The nutrition transition in developing Asia: Dietary change, drivers and health impacts. In P. Jackson, W. Spiess \& F. Sultana (Eds.), Eating, drinking: surviving (Springer Briefs in Global Understanding). Cham: Springer. http://dx.doi.org/10.1007/978-3-319-42468-2_9. 
Percepção sobre mídia e comportamento na compra de alimentos: estudo com consumidores de dois municípios do sul de Minas

Gerais

Carvalho, G. R. et al.

Maia, E. G., Costa, B. V. L., Coelho, F. S., Guimarães, J. S., Fortaleza, R. G., \& Claro, R. M. (2017). Análise da publicidade televisiva de alimentos no contexto das recomendações do Guia Alimentar para a População Brasileira. Cadernos de Saúde Pública, 33(4), e00209115. PMid:28538798. http://dx.doi.org/10.1590/0102-311x00209115

Marzarotto, B., \& Alves, M. K. (2017). Leitura de rótulos de alimentos por frequentadores de um estabelecimento comercial. Ciência \& Saúde, 10(2), 102-108. http://dx.doi.org/10.15448/1983-652X.2017.2.24220

Mayhew, A. J., Lock, K., Kelishadi, R., Swaminathan, S., Marcilio, C. S., Iqbal, R., Dehghan, M., Yusuf, S., \& Chow, C. K. (2016). Nutrition labelling, marketing techniques, nutrition claims and health claims on chip and biscuit packages from sixteen countries. Public Health Nutrition, 19(6), 998-1007. PMid:25818889. http://dx.doi.org/10.1017/S1368980015000658

Mills, S. D., Tanner, L. M., \& Adams, J. (2013). Systematic literature review of the effects of food and drink advertising on food and drink-related behaviour, attitudes and beliefs in adult populations. Obesity Reviews, 14(4), 303-314. PMid:23297736. http://dx.doi.org/10.1111/obr.12012

Nestle, M., \& Ludwig, D. S. (2010). Front-of-package food labels: Public health or propaganda? Journal of the American Medical Association, 303(8), 771-772. PMid:20179287. http://dx.doi.org/10.1001/jama.2010.179

Programa das Nações Unidas para o Desenvolvimento - UNDP. (2017). Desenvolvimento Humano e IDH. Recuperado em 30 de julho de 2018, de http://www.br.undp.org/content/brazil/pt/home/idh0.html

Sadeghirad, B., Duhaney, T., Motaghipisheh, N. R., Campbell, N. R., \& Johnston, B. C. (2016). Influence of unhealthy food and beverage marketing on children's dietary intake and preference: A systematic review and meta-analysis of randomized trials. Obesity Reviews, 17(10), 945-959. PMid:27427474. http://dx.doi.org/10.1111/obr.12445

Sainsbury, E., Colagiuri, S., \& Magnusson, R. (2017). An audit of food and beverage advertising on the Sydney metropolitan train network: Regulation and policy implications. BMC Public Health, 17(1), 490-501. PMid:28532472. http://dx.doi.org/10.1186/s12889-017-4433-2

Financiamento: Nenhum. 